\title{
YopD and LcrH Regulate Expression of Yersinia enterocolitica YopQ by a Posttranscriptional Mechanism and Bind to yopQ RNA
}

\author{
Deborah M. Anderson, $\uparrow$ Kumaran S. Ramamurthi, $\ddagger$ Christina Tam, $\ddagger$ \\ and Olaf Schneewind * $^{*}$ \\ Department of Microbiology \& Immunology, University of California-Los Angeles \\ School of Medicine, Los Angeles, California 90095
}

Received 14 September 2001/Accepted 16 November 2001

\begin{abstract}
Pathogenic yersiniae secrete 14 Yop proteins via the type III pathway. Synthesis of YopQ occurs when the type III machinery is activated by a low-calcium signal, but not when the calcium concentration is above 100 $\mu M$. To characterize the mechanism that regulates the expression of yop $Q$, mutants that permit synthesis of YopQ in the presence of calcium were isolated. Yersiniae bearing deletion mutations in yopN, tyeA, $\operatorname{sycN}$, or yscB synthesized and secreted YopQ in both the presence and the absence of calcium. In contrast, yersiniae with a deletion in yopD or $\mathrm{lcrH}$ synthesized YopQ in the presence of calcium but did not secrete the polypeptide. These variants displayed no defect in YopQ secretion under low-calcium conditions, revealing that yopD and $l c r H$ are required for the regulation of $y o p Q$ expression. Experiments with transcriptional and translational fusions to the $n p t$ reporter gene suggest that yopD and $l c r H$ regulate yop $Q$ expression at a posttranscriptional step. YopD and LcrH form a complex in the bacterial cytosol and bind yop $Q$ mRNA. Models that can account for posttranscriptional regulatory mechanisms of yop expression are discussed.
\end{abstract}

Three pathogenic Yersinia species, Y. pestis, Y. enterocolitica, and $Y$. pseudotuberculosis, use a type III secretion machinery to transport Yop proteins across the bacterial double membrane envelope $(16,43)$. The transport of Yop proteins plays an important role during the establishment of bacterial infections, as Yersinia spp. lacking type III genes are rapidly phagocytosed and killed by host macrophages $(21,24,51,55)$. The genes that encode the type III secretion machinery and Yop proteins are located on the $70-\mathrm{kb}$ virulence plasmid of yersiniae (17). $Y$. enterocolitica yopB, yopD, yopE, yop $H$, yopM, yopN, yopO, yopP, yop $Q, y o p R, y o p T, l c r V, y s c M 1$, and yscM2 encode secretion substrates. $Y$. pseudotuberculosis and $Y$. pestis virulence plasmids encode only yscM1 (lcrQ) but not yscM2 (58, 61). yscC, $y s c D, y s c E, y s c F, y s c G, y s c I, y s c J, y s c K, y s c L, y s c N, y s c O, y s c P$, $y s c Q, y s c R, y s c S, y s c T, y s c U, y s c V, y s c W, y s c X$, and $y s c Y$ specify components of the type III secretion machinery $(1,2,7)$.

During Yersinia infection of tissue culture cells, the type III machinery is activated by a series of environmental signals that trigger the transport of specific sets of Yop proteins (42). A temperature shift to $37^{\circ} \mathrm{C}$ and an environmental glutamate signal lead to the assembly and activation of the type III machinery $(31,42,71)$. In the presence of additional signals, i.e., animal serum proteins, $Y$. enterocolitica secretes YopB, YopD, YopR, and LcrV into the extracellular medium (42, 44, 45).

\footnotetext{
* Corresponding author. Mailing address: Committee on Microbiology, The University of Chicago, 920 East 58th Street, Chicago, IL 60637. Phone: (773) 834-9060. Fax: (773) 834-8150. E-mail: oschnee @ delphi.bsd.uchicago.edu.

$\dagger$ Present address: Division of Cellular and Molecular Medicine, School of Medicine, University of California San Diego, La Jolla, CA 92093.

$\$$ Present address: Committee on Microbiology, The University of Chicago, Chicago, IL 60637.
}

Contact with host cells activates the type III machinery to transport YopE, YopH, YopM, YopN, YopO, YopP, YopT, and YscM1 into the eukaryotic cytosol (9, 10, 28, 36, 41, 49, 50, $52,59)$. Our laboratory refers to these transport reactions as type III secretion (YopB, YopD, YopR, and LcrV) and type III targeting (YopE, YopH, YopM, YopN, YopO, YopP, YopT, and YscM1) (41).

Bacterial type III machines assemble into needle complexes, supramolecular structures that are dedicated to protein transport (40). Upon contacting tissue culture cells, the needle complexes of Yersinia type III machines insert into the plasma membrane of target cells and serve as protein conduits for the transport of YopE, YopH, YopM, YopN, YopO, YopP, YopT, and YscM1 (32). Needles are presumably also involved in measuring environmental signals, as the needle insertion into host cells could provide the low-calcium signal of the Yersinia type III pathway (42).

Some of the views presented here are under debate. Although it seems clear that YopB and YopD are predominantly secreted proteins, portions of the polypeptides are associated with the plasma membrane of eukaryotic cells $(41,65)$. WolfWatz, Cornelis, and colleagues postulated that YopB, YopD, and LcrV form a pore in the host cell plasma membrane through which effector Yops (YopE, YopH, YopM, YopO, YopP, and YopT) are translocated into the cytosol (19, 29, 33, $65)$. This translocation mechanism is obviously distinct from protein transport through the conduit of type III needles, which do not appear to contain YopB, YopD, and LcrV (32). Another area of debate is the precise location of LcrV. Although LcrV is regularly found in the extracellular medium (45), this polypeptide has also been observed on the surface of yersiniae (53) or transported into the cytosol of tissue culture cells (22). 
The mechanisms by which Yop proteins are recognized by the type III machinery are currently under investigation by several laboratories. Translational fusion of yop genes to the $5^{\prime}$ end of the Escherichia coli neomycin phosphotransferase (npt) or Bordetella pertussis adenylate cyclase (cya) gene results in the type III secretion of hybrid polypeptides $(4,60)$. As for yop $Q$, truncation of $3^{\prime}$ coding sequences is tolerated, and fusion of only the first 15 codons to $n p t$ is still sufficient to promote type III secretion of hybrid polypeptides (6). Deletion of codons 2 to 15 of yop $Q$ abrogated substrate recognition, indicating that the signal encoded in the first 15 codons is indeed necessary for type III transport of hybrid polypeptides (6). Anderson et al. generated nucleotide insertions and deletions of the yop $Q$, yopN, and yopE secretion signal, immediately following the AUG start codon $(4,6)$. These frameshift mutations were corrected by reciprocal insertions and deletions at the fusion site between yop $Q$ and $n p t$, allowing the synthesis of hybrid Npt proteins (4). Some but not all of the frameshifted signals directed mutant proteins into the type III pathway. Anderson et al. suggested that the secretion signal of Yop proteins may be decoded at the level of mRNA translation rather than via signal peptide recognition by the type III secretion machinery (5).

Some Yop proteins, for example YopE, bind to small homodimeric cytoplasmic proteins (Syc, specific Yop chaperone) (68). In the case of YopE, this interaction also leads to the initiation of the polypeptide into the type III pathway $(12,15)$. Nevertheless, this mechanism may not be universal, as Syc proteins have not yet been described for YopM, YopO, YopP, and YopQ. What is the role of Syc proteins in directing Yops into the type III pathway? Cornelis and colleagues proposed that Syc proteins could act as secretion chaperones or transport pilots (66). In this model, Syc proteins deliver Yops to the type III machinery and may be involved in substrate recognition (69). Karlinsey et al. suggested that Syc proteins may act as modulators of translation of secretion substrates, presumably coupling translation of mRNA with the type III secretion of newly synthesized polypeptides (39). Lloyd et al. proposed the existence of a signal peptide within the first 12 amino acid residues (46). This signal peptide is predominantly composed of alternating polar and hydrophobic residues. Furthermore, a synthetic secretion signal seems functional, as the peptide sequence MSISISISI can direct YopE into the type III pathway (47). In the signal peptide model, Syc proteins may prevent the premature folding or association of secretion substrates (46). Future work will need to distinguish between these models and reveal the mechanism by which Yop proteins are recognized and transported by the type III pathway.

In this report we have focused on the regulation of expression of type III secretion substrates. Synthesis of YopQ occurs when the type III pathway is activated by an environmental calcium signal $(\leq 80 \mu \mathrm{M})$, but not when the calcium concentration is above $100 \mu \mathrm{M}$ (6). We sought to characterize mutants that permit synthesis of YopQ in the presence of calcium. As reported previously, mutants bearing deletion mutations in yop $N$, tye $A$, $s y c N$, or $y s c B$ display a calcium-blind phenotype and secrete Yops in the presence and absence of calcium (14, $20,38,72)$. We refer to these strains as class I mutants, as the Yersinia variants synthesize and secrete YopQ in the presence and in the absence of calcium. Mutants with a deletion in yopD or $l c r H$ synthesize YopQ in the presence of calcium but do not secrete the polypeptide. These class II mutants display no defect in YopQ secretion, revealing that yopD and $l c r H$ are required for the regulation of yop $Q$ expression. Experiments with transcriptional and translational yop $Q$ fusions to the $n p t$ reporter gene suggest that yopD and $l c r H$ may regulate yop $Q$ expression at a posttranscriptional step. YopD and LcrH form a complex in the bacterial cytosol and bind yop $Q$ mRNA.

\section{MATERIALS AND METHODS}

Bacterial strains and growth conditions. Y. enterocolitica W22703 (18), MC1 $[\Delta(y o p Q)](6), \operatorname{MC} 4[\Delta(y o p B)](44), \operatorname{VTL} 1[\Delta(y o p N)](41)$, and VTL2 $[\Delta(y o p D)]$ (44) have been described previously. Using allelic exchange, codons 21 to 149 of $l c r H$ were replaced with GGA TCC, generating the $\Delta(l c r H)$ strain CT133. The $\triangle(y o p D Q)$ strain DA1 was constructed by crossing the yop $Q 1$ mutation into the yopD mutant strain VTL2. All other mutations were introduced by allelic exchange using an established strategy (12). E. coli strain P90C served as the host for DNA manipulations (48).

DNA methods and plasmid construction. Plasmid pDA255, expressing gstyopD under control of $l a c I^{\mathrm{q}}$ from the tac promoter, has been described previously (41). The yopB and $l c r H$ open reading frames were PCR amplified using the primers YopB-Kpn (5'-AAGGTACCCAACAAGAGACGACAGACA-3') and YopB-Bam $($ yop $B)(41)$ or LcrH-Kpn (5'-AAGTACCCAACAAGAGACGAC AGACA- $\left.3^{\prime}\right)$ and LcrH-Bam (5'-AAGGATCCTCATGGGTTATCAACGCAC T-3') $(l c r H)$. DNA fragments were cut with $K p n I$ and Bam HI and cloned into the corresponding sites of pDA255, generating pNT20 (gst-yopB) and pDA326 (gst$l c r H$ ). Plasmid pDA325 was generated by PCR amplification with the primers LcrH-Nde (5'-AACATATGCAACAAGAGACGACAGA-3') and LcrH-Bam. The PCR product was cut with $N d e \mathrm{I}$ and $B a m \mathrm{HI}$ and cloned into the corresponding site of pVL41 (41). An $n p t$ reporter cassette that allows fusion of yop $Q$ sequences has been described previously (pDA183 and pDA243) (6).

To generate pDA330, npt sequences were PCR amplified with the primers Npt-Tsf (5'-AAGGTACCTGACTGACTGATCAAGAGACAGGATGAGGA T-3') and Npt-3 (6), and the yopQ promoter was amplified with primers YopQ-1 (5'-AAGAATTCAGCCATTATTTTGCTATACCGA-3') and YopQ-TS + 1 (5'AAGGTACCATTATTTATTTTAAAGCTACTGAT-3'). Amplified fragments were cut with EcoRI and KpnI and KpnI and NdeI and cloned into pDA183. Plasmid pDA340 was generated by PCR amplification of yop $Q$ sequences with the primers YopQ-T7 (5'-AAGACGGTTATTAAATAGTGTAG-3') and Npt-3

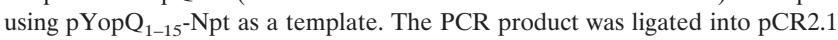
(Invitrogen). pDA183 was generated by cloning a yop $Q$ promoter and yop $Q$ untranslated region (UTR) fusion to the $n p t$ reporter gene into the pHSG575 derivative pDA15 $(12,64)$.

pDA243 is a pHSG575 derivative harboring an insert in which the yop $Q$ promoter, yop $Q \mathrm{UTR}$, and full-length open reading frame are fused to the open reading frame of $n p t$. The pHSG575 derivative pDA209 contains a transcriptional fusion of the yop $Q$ promoter, yop $Q$ UTR, and yop $Q$ full-length open reading frame to the open reading frame of $n p t$. Translational initiation of $n p t$ in pDA209 occurs from its own regulatory signals.

Protein electrophoresis and immunodetection. Procedures to measure Yop secretion have been reported previously (4). To quantify the concentration of Npt fusions in the presence of calcium in Fig. 2, wild-type and mutant Yersinia cultures were induced by temperature shift in the presence of calcium. One milliliter of culture was removed and precipitated with trichloroacetic acid (TCA). Equal amounts of protein, as determined by the cultures' absorbance at $600 \mathrm{~nm}$, were loaded on sodium dodecyl sulfate-polyacrylamide gel electrophoresis (SDS-PAGE), followed by electrotransfer to polyvinylidene difluoride membranes and immunoblotting. The signal was visualized by incubation of the membrane with [ $\left.{ }^{125} \mathrm{I}\right]$ protein A and quantified on a PhosphorImager. ysc genes were expressed as His-tagged fusions in E. coli. Recombinant Ysc proteins were purified by affinity chromatography and injected into rabbits to raise the Yscspecific antisera used (see Table 1).

Purification of GST fusion proteins. Yersinia expressing glutathione $S$-transferase (GST)-YopB, GST-YopD, or GST-LcrH were grown in tryptic soy broth (TSB) at $37^{\circ} \mathrm{C}$ for $3 \mathrm{~h}$ in the presence of $5 \mathrm{mM} \mathrm{CaCl} \mathrm{Cr}_{2}$ or $5 \mathrm{mM}$ EGTA. Thirty minutes after beginning incubation at $37^{\circ} \mathrm{C}$, the expression of GST fusions was induced by the addition of $1 \mathrm{mM}$ IPTG (isopropylthiogalactopyranoside). Cells were harvested by centrifugation, suspended in 10 mM HEPES ( $\mathrm{pH} 7.5)-100$ $\mathrm{mM}$ potassium acetate $-2 \mathrm{mM}$ magnesium chloride $-1 \mathrm{mM}$ dithiothreitol (DTT) and lysed in the presence of $50 \mu \mathrm{M}$ phenylmethylsulfonyl fluoride at $10,000 \mathrm{lb} / \mathrm{in}^{2}$ 
in a French pressure cell. The lysate was cleared by centrifugation at $15,000 \times g$ for $15 \mathrm{~min}$ and applied to affinity chromatography. Glutathione-Sepharose, 1.5 -ml bed volume, was equilibrated with $50 \mathrm{mM}$ Tris-HCl- $150 \mathrm{mM} \mathrm{NaCl}, \mathrm{pH}$ 7.5 , and charged with lysate. The column was washed with $30 \mathrm{ml}$ of $50 \mathrm{mM}$ Tris-HCl- $150 \mathrm{mM} \mathrm{NaCl}-10 \%$ glycerol, $\mathrm{pH} 7.5$, and bound proteins were eluted with $10 \mathrm{mM}$ glutathione in $50 \mathrm{mM}$ Tris- $\mathrm{HCl}, \mathrm{pH} 8.0$.

Reverse transcriptase PCR. Yersinia cultures were grown at $37^{\circ} \mathrm{C}$ in the presence or absence of calcium ions for $2 \mathrm{~h}$. Total RNA was isolated as described previously (6), and $5 \mu \mathrm{g}$ of RNA was treated with $2 \mathrm{U}$ of DNase I for $30 \mathrm{~min}$ at $37^{\circ} \mathrm{C}$, followed by phenol extraction and ethanol precipitation. Each sample was divided into three tubes, and cDNA was prepared. The oligonucleotides Npt-3 (6) or Cat-2 (5'-AAGGATCCAAATTACGCCCCGCCCTG-3') were annealed to RNA by heating to $37^{\circ} \mathrm{C}$ for $5 \mathrm{~min}$, followed by a 5 -min ice incubation in reverse transcriptase buffer. Avian myeloblastosis virus (AMV) reverse transcriptase (Promega) was added together with $0.5 \mathrm{mM}$ deoxynucleoside triphosphates and incubated at $42^{\circ} \mathrm{C}$ for $60 \mathrm{~min}$. Reactions were phenol extracted, and the DNA was ethanol precipitated and suspended in $40 \mu \mathrm{l}$ of $\mathrm{H}_{2} \mathrm{O}$. The cDNA was used as the template for PCR amplification with three sets of primers: YopQ+1/ Npt-3, Npt-1/Npt-3 (4, 6), and Cat-1 (5'-AAGGTACCGAGAAAAAAATCAC TGGATATA-3')/Cat-2. PCR products were separated by electrophoresis on agarose gels.

RNA electrophoretic mobility shift assay. yopD, $l c r H$, and a transcriptional yopD-lcrH fusion were cloned into pET16 (Novagen). Recombinant plasmids pKR2 (yopD), pKR4 (lcrH), and pKR6 (yopD-lcrH) were transformed into E. coli BL21(DE3) (63). His 6 affinity-tagged proteins were expressed by $\mathrm{T} 7$ polymerase induction of E. coli cultures with IPTG (63). E. coli cells ( $10^{12}$ cells) were lysed in a French press at $18,000 \mathrm{lb} / \mathrm{in}^{2}$ (total extract), and insoluble material was removed at $115,000 \times g$. The supernatant (load) was applied to Ni-nitrilotriacetic acid (NTA) preequilibrated with $0.05 \mathrm{M}$ Tris- $\mathrm{HCl}-0.15 \mathrm{M} \mathrm{NaCl}, \mathrm{pH}$ 7.5. The column was washed with the same buffer containing 0.02 to $0.07 \mathrm{M}$ imidazole and eluted with $0.5 \mathrm{M}$ imidazole.

An RNA probe ( -45 through +45 relative to the AUG of yop $Q$ ) was synthesized in vitro using T7 RNA polymerase and $\left[\alpha-{ }^{32} \mathrm{P}\right] \mathrm{UTP}$. RNA was heated at $95^{\circ} \mathrm{C}$ for $2 \mathrm{~min}$ and cooled to $4^{\circ} \mathrm{C}$ for $10 \mathrm{~min}$. Purified protein and $10 \mathrm{fmol}$ of yopQ RNA were incubated in $25 \mathrm{mM}$ HEPES- $150 \mathrm{mM} \mathrm{KCl}-10 \mathrm{mM} \mathrm{MgCl} \mathrm{M}_{2}-$ $1 \mathrm{mM}$ DTT-1\% glycerol-40 U of RNasin (Promega), $\mathrm{pH} 7.5$, on ice for $10 \mathrm{~min}$ prior to separation by electrophoresis on a $4 \%$ polyacrylamide gel. Competition experiments used unlabeled yop $Q$ mRNA and $E$. coli tRNA.

\section{RESULTS}

Genes required for yop $Q$ expression. When the type III machinery is induced by the chelation of calcium ions, yersiniae synthesize and secrete YopQ into the extracellular medium (6). To identify the genes that are required for the regulation of yop $Q$ expression, we generated nonpolar mutations in the $Y$. enterocolitica W22703 virulence plasmid using an allelic exchange strategy (12). The Yersinia virulence plasmid carries 22 $y s c$ genes (ysc $A, y s c C, y s c D, y s c E, y s c F, y s c G, y s c I, y s c J, y s c K$, $y s c L, y s c N, y s c O, y s c P, y s c Q, y s c R, y s c S, y s c T, y s c U, y s c V, y s c W$, $y s c X$, and $y s c Y$ ) that specify secretion machinery components and 10 regulatory genes $(l c r G, l c r V, l c r H, s y c N, s y c H, y o p D$, $y o p N$, yscB, yscM1, and yscM2) that control the activity of the type III pathway (17). Yersinia strains harboring virulence plasmids with null mutations in any one of those genes were analyzed for YopQ synthesis and secretion. Three mutant phenotypes were detected: loss of calcium regulation of YopQ synthesis and secretion (class I); loss of calcium regulation of YopQ synthesis but not of type III secretion (class II); and loss of YopQ synthesis under low-calcium conditions (class III) (Fig. 1A).

The products of class I genes (yopN, tye $A$, $\operatorname{sycN}$, and yscB) are thought to act by occluding the secretion channel $(13,72)$. Synthesis and secretion of YopQ in these mutants occur even in the presence of calcium. Class II genes (yopD and $l \mathrm{crH}$ ) appear to specify regulatory proteins that prevent the expression of yop $Q$ in the presence of calcium (see below). Class III

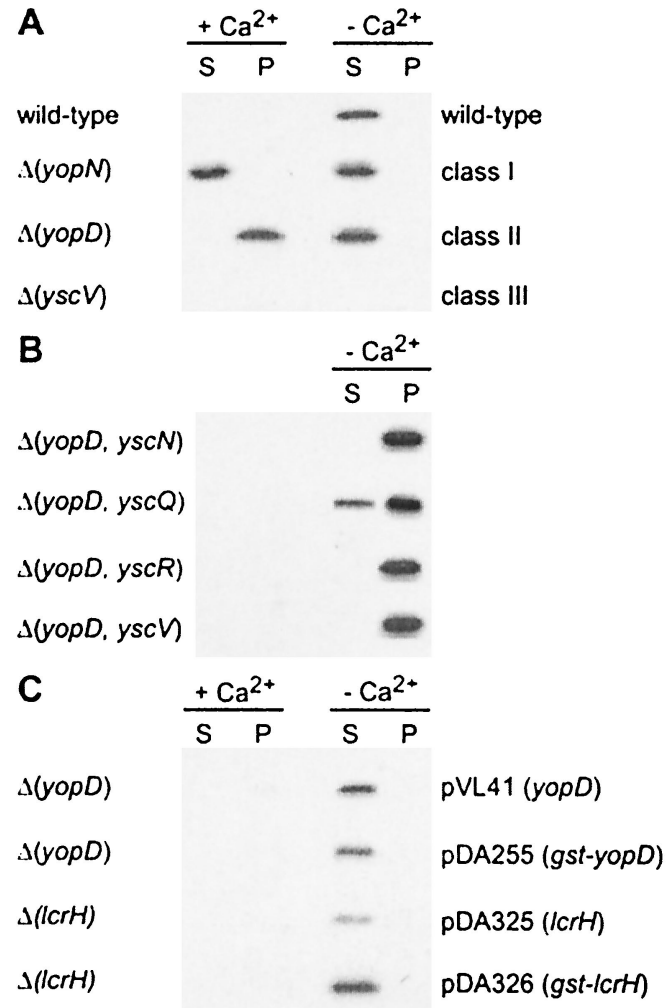

FIG. 1. Synthesis and type III secretion of YopQ in wild-type and mutant yersiniae. (A) Y. enterocolitica W22703 (wild type) and isogenic mutant strains VTL1 $[\Delta(y o p N)]$, VTL2 $[\Delta(y o p D)]$, and KUM1 $[\Delta(y s c V)]$ were grown in the presence and absence of calcium (low calcium is an inducing condition for type III secretion). Cultures were centrifuged, and proteins secreted into the culture medium (S, supernatant) were separated from the cell sediment (P, pellet). YopQ secretion was measured by immunoblotting with specific antiserum. (B) $Y$. enterocolitica strains W22703 (wild type), VTL2 [ $\Delta(y o p D)]$, and CT133 $[\Delta(l c r H)]$ were grown at $37^{\circ} \mathrm{C}$ in the presence and absence of calcium. Culture supernatants $(\mathrm{S})$ and bacterial extracts $(\mathrm{P})$ were separated on SDS-PAGE and analyzed by immunoblotting with specific antiserum $(\alpha$-YopQ). Yersinia strains were transformed with plasmids encoding wild-type yopD (pVL41) (44), gst-yopD (pDA255) (44), lcrH (pDA325), or gst-lcrH (pDA326). (C) YopQ secretion in Y. enterocolitica strains carrying knockout mutations in both the yopD and ysc genes.

genes encode components of the secretion machinery (ysc $A$, $y s c C, y s c D, y s c E, y s c F, y s c G, y s c I, y s c J, y s c K, y s c L, y s c N, y s c O$, $y s c P, y s c Q, y s c R, y s c S, y s c T, y s c U, y s c V, y s c W, y s c X$. and $y s c Y)$, and knockout mutations in these genes abrogate the expression of yopQ (6). YscM1 (LcrQ) and YscM2, factors that are thought to control yop transcription, were not considered for the regulation of yop $Q$ translation (61).

Genes required for YopQ secretion. E. coli expressing the secretion genes of the plant pathogen Erwinia chrysanthemi export YopQ into the extracellular medium (3). The type III machinery of $E$. chrysanthemi is encoded by 11 hrc genes, all of which display homology to Yersinia ysc genes (yscC, yscD, yscJ, $y s c L, y s c N, y s c Q, y s c R, y s c S, y s c T, y s c U$, and $y s c V$ ) (30). These 11 ysc genes are conserved among type III machines of other gram-negative bacteria, and 9 genes, $y s c D, y s c L, y s c N$, ysc $Q$, $y s c R, y s c S, y s c T, y s c U$, and $y s c V$, are also present in flagellar type III systems (34). To assess whether $y s c D, y s c L, y s c N$, ysc $Q$, $y s c R, y s c S, y s c T, y s c U$, and $y s c V$ are required for the secretion 
TABLE 1. Yersinia type III machinery components and their role in YopQ secretion

\begin{tabular}{lrc}
\hline $\begin{array}{c}\text { Type III } \\
\text { protein }\end{array}$ & $\begin{array}{c}\text { Ysc solubility }^{a} \\
(\%)\end{array}$ & $\begin{array}{c}\text { YopQ secretion by } \Delta(y s c, y o p D) \\
\text { mutant }^{b}(\% \text { of control })\end{array}$ \\
\hline YscD & 0 & 70 \\
YscL & 100 & 0 \\
YscN & 50 & 0 \\
YscQ & 100 & 20 \\
YscR & 0 & 0 \\
YscS & 0 & 0 \\
YscT & NT & 0 \\
YscU & 0 & 0 \\
YscV & 0 & 0 \\
Control & NA & 100 \\
\hline
\end{tabular}

${ }^{a}$ Yersiniae were lysed by French press, and crude extracts were centrifuged $(100,000 \times g)$. Supernatant and sediment were separated and analyzed by immunoblotting with specific antisera. The amount of immuno reactive Ysc in the supernatant is shown. NT, not tested; NA, not applicable.

${ }^{b}$ YopQ secretion in low-calcium-induced Yersinia cultures was measured by immunoblotting. Y. enterocolitica VTL2 (ysc wild type) $\Delta(y o p D)$ secreted $100 \%$ YopQ, whereas $\Delta(y s c, y o p D)$ mutants displayed the secretion defects recorded.

of YopQ, the yopD knockout allele was combined with various ysc mutations, generating mutant strains capable of synthesizing YopQ (Fig. 1B).

$\Delta($ yscL/yopD $), \Delta(y s c N / y o p D), \Delta(y s c R / y o p D), \Delta(y s c S / y o p D)$, $\Delta(y s c T / y o p D), \Delta(y s c U / y o p D)$, and $\Delta(y s c V / y o p D)$ strains were completely defective in the type III secretion of YopQ, and mutants $\Delta(y s c Q / y o p D)$ and $\Delta(y s c D / y o p D)$ displayed significant defects in YopQ secretion (Table 1). Thus, all nine genes, $y s c D, y s c L, y s c N, y s c Q, y s c R, y s c S, y s c T, y s c U$, and $y s c V$, are required for the efficient secretion of YopQ. To determine the subcellular locations of YscD, YscL, YscN, YscQ, YscR, YscS, and YscU, crude Yersinia extracts were subjected to ultracentrifugation at $100,000 \times g$, sedimenting bacterial membranes. YscD, YscR, YscS, YscU, and YscV were found in the sediment of centrifuged samples, whereas YscL, YscQ, and some YscN remained soluble in the cytoplasmic supernatant. Together these results suggest that the Yersinia type III machine is composed of cytoplasmic and membrane components, with YscN representing a mobile machinery protein.
LcrH and YopD regulate yop $Q$ expression. Class II mutant strains, $\Delta(y o p D)$ and $\Delta(l c r H)$, fail to prevent yop $Q$ expression in the presence of calcium. To determine whether the mutant phenotype can be complemented in trans, $Y$. enterocolitica VTL2 $[\Delta(y o p D)]$ and CT133 $[\Delta(l c r H)]$ were transformed with plasmids containing wild-type alleles. When introduced into the corresponding mutant strain, plasmid-encoded yopD or $l c r H$ prevented yop $Q$ expression in the presence of calcium (Fig. 1C). Previous work showed that YopD is exported by the type III pathway (27). LcrH (SycD) binds to YopD and YopB in the bacterial cytoplasm and functions as a chaperone for the secretion of these polypeptides (67). Fusion of YopD to the C terminus of GST abolishes type III secretion of the hybrid protein, causing GST-YopD to reside in the bacterial cytoplasm (44).

We asked whether the defect of the $\Delta($ yopD $)$ strain in yop $Q$ regulation would be complemented by GST-YopD. Plasmidencoded GST-YopD but not GST-LcrH restored the calcium regulation of yop $Q$ expression in the $\Delta(y o p D)$ strain (Fig. $1 \mathrm{C}$ and data not shown). Similarly, the regulatory defect of the $\Delta(l c r H)$ strain was complemented by GST-LcrH but not by GST-YopD (Fig. 1C and data not shown). Thus, LcrH and YopD each perform an essential function in regulating the expression of yop $Q$ in the cytoplasm of $Y$. enterocolitica.

YopD and LcrH regulate yop $Q$ expression by a posttranscriptional mechanism. To examine the regulatory defect of yopD and $l c r H$ mutant yersiniae, yop $Q$ sequences were fused to the $5^{\prime}$ end of the $n p t$ open reading frame (57). Bacteria were grown at $37^{\circ} \mathrm{C}$ in the presence of calcium, and the concentration of Npt protein in bacterial extracts was measured by immunoblotting (Fig. 2). The data are presented as the ratio of Npt expression between mutant and wild-type cells. Fusion of the yop $Q$ promoter to $n p t$ revealed that the yopD and $l c r H$ mutant strains caused only a modest increase in transcription of the reporter gene (1.5- to 2-fold). However, fusion of yop $Q$ translational initiation signals, i.e., the promoter and $5^{\prime}$ untranslated leader of yop $Q$ to $n p t$, detected a defect in posttranscriptional regulation, as $\Delta(y o p D)$ and $\Delta(l c r H)$ yersiniae increased expression by five- to sixfold. Fusion of the entire yop $Q$
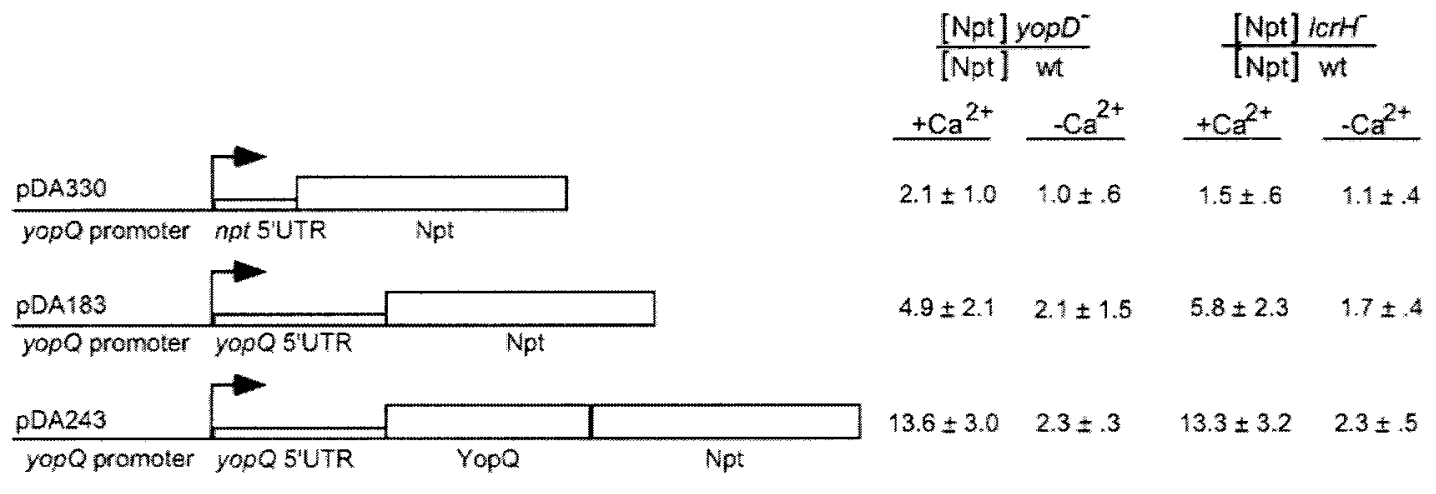

FIG. 2. $l c r H$ and yopD regulate $y o p Q$ expression at a posttranscriptional step. Expression of yop $Q$ was measured by fusing the reading frame of $n p t$ to either the yopQ promoter (pDA330), the promoter and $5^{\prime}$ UTR (pDA183), or the $3^{\prime}$ end of the yopQ reading frame specifying a translational YopQ-Npt fusion (pDA243). Plasmids were transformed into $Y$. enterocolitica W22703 (wild type) or the isogenic $\Delta($ lcrH) (CT133) and $\Delta(y o p D)$ (VTL2) mutant strains. Yersinia strains were grown in the presence or absence of calcium, and bacterial extracts were prepared by TCA precipitation. The concentration of Npt reporter was determined by immunoblotting and is reported as the ratio between mutant and wild-type cells. Data were averaged from more than three independent experiments. The standard deviation $( \pm)$ is indicated. 

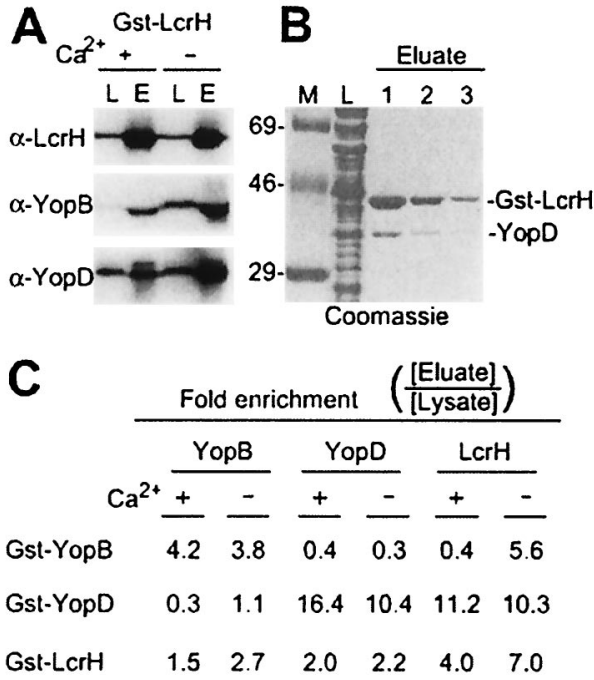

FIG. 3. GST-LcrH binds to YopD or YopB. Plasmid pDA326 was transformed into the $\Delta(l c r H)$ mutant Yersinia strain CT133, and the expression of GST-LcrH was induced by the addition of IPTG. Bacterial lysates were cleared by centrifugation, and GST-LcrH was purified by affinity chromatography on glutathione-Sepharose. (A) Purification was assessed on Coomassie-stained SDS-PAGE and by immunoblotting of load and eluate fractions. (B) When expressed in $\Delta(y o p D)$ cells, subjected to affinity chromatography, and measured on Coomassie-stained SDS-PAGE, GST-YopD bound to LcrH but not to YopB (VTL2 harboring pDA255). Molecular size markers (lane M, in kilodaltons), lysate (L), and eluate fractions (1,2, and 3) are indicated. (C) Purification of GST-YopB, GST-YopD, and GST-LcrH was measured by subjecting affinity chromatography load (lysate) and eluate fractions to immunoblotting. Chemiluminescent signals were scanned and quantified and are reported as the ratio of signal intensity between eluate and lysate. This experiment was performed in duplicate, with yersiniae grown in the presence and absence of calcium.

gene to npt caused an even greater (13- to 14-fold) increase in reporter gene expression. Together these data suggest that YopD and LcrH regulate the expression of yop $Q$ at a posttranscriptional step.

LcrH binds YopB and YopD in the bacterial cytoplasm. Previous work reported LcrH (SycD) binding to YopB and YopD (66). These studies involved the copurification of overexpressed proteins in E. coli or the binding of LcrH to YopD immobilized on a nitrocellulose filter. To measure the binding of LcrH to YopB or YopD in the Yersinia cytoplasm, GST fusions to YopB, YopD, and LcrH were expressed from the IPTG-inducible tac promoter. Bacterial extracts were subjected to affinity chromatography, and copurification of polypeptides was measured by immunoblotting.

As expected, both YopB and YopD copurified with GSTLcrH. GST-YopB and GST-YopD each copurified with LcrH but not with one another (Fig. 3). Purification of GST-YopB from the cytoplasm of the $\Delta(l c r H)$ mutant strain was greatly increased when yersiniae were grown in the absence rather than in the presence of calcium. For all other purifications, the growth of yersiniae in the presence or absence of calcium did not significantly alter the purification profiles. Thus, LcrH binds to either YopB or YopD to form cytoplasmic bipartite complexes (66), whereas YopD does not appear to bind to YopB. Consistent with this interpretation of the data is our observation that $\Delta(l c r H)$ and $\Delta($ yop $D)$ but not $\Delta($ yopB $)$ strains are defective in repressing YopQ synthesis (data not shown).

YopD/LcrH are required for the degradation of $y o p Q$ mRNA. Posttranscriptional control of gene expression can occur as a block in translation (25) and/or as degradation of mRNA (26). Synthesis of a plasmid-encoded transcript encompassing both the $y o p Q$ and $n p t$ open reading frames (6) was induced by temperature shift in the $\Delta(y o p Q)$ Yersinia strain MC3 (Fig. 4). $n p t$ was expressed in both the presence and absence of calcium, whereas yop $Q$ was expressed and YopQ polypeptide was secreted only in the absence of calcium (Fig. 4) (6). Total RNA
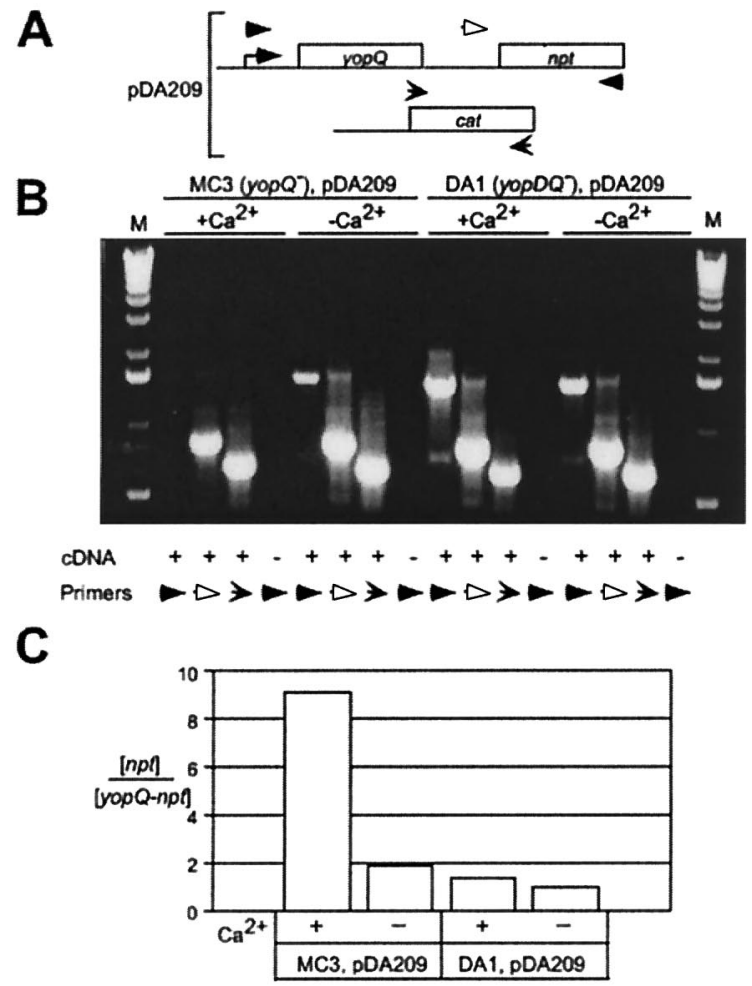

D

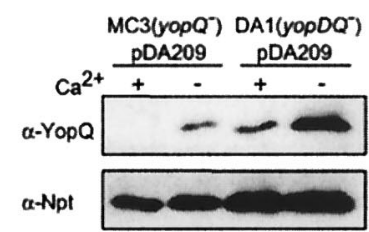

FIG. 4. YopD is required for degradation of yop $Q$ mRNA. (A) Plasmid pDA209 carries the cat gene and a transcriptional yopQ-npt fusion. The drawing displays the annealing positions of oligonucleotide primers (arrowheads). (B) Y. enterocolitica strains MC3 $[\Delta($ yop $Q)]$ and DA1 $[\Delta(y o p D Q)]$ were transformed with pDA209 (6) and grown in either the presence or absence of calcium. RNA was purified and cDNA was synthesized ( + and - indicate the addition or omission of AMV reverse transcriptase, respectively) using oligonucleotides that anneal at the $3^{\prime}$ end of yopQ-npt or cat. cDNA template was used for PCR amplification (arrowheads), and products were analyzed on ethidium bromide-stained agarose gel. The 1-kb DNA ladder was used for size calibration (lane M). (C) The ratio of $n p t / y o p Q$ to $n p t$ transcripts in various strains and growth conditions was determined by quantifying fluorescent signals. (D) Cell extracts of $Y$. enterocolitica MC3(pDA209) and DA1(pDA209) were analyzed by immunoblotting with $\alpha$-YopQ and $\alpha$-Npt. 
was isolated, and cDNA was synthesized using reverse transcriptase and an oligonucleotide annealing to the $3^{\prime}$ end of $n p t$. The cDNA was PCR amplified using the same $3^{\prime}$ primer as well as $5^{\prime}$ primers annealing to either the transcriptional start site of yop $Q$ or the ribosome-binding site of $n p t$.

In the absence of calcium (when YopQ is expressed), PCR amplification detected similar amounts of full-length yopQ-npt transcript as well as $3^{\prime} n p t$ mRNA sequence. The addition of calcium to the culture medium not only blocked type III secretion but also reduced the concentration of the full-length yopQ-npt transcript. In contrast, the amount of the $3^{\prime} n p t$ portion of the yopQ-npt transcript was not reduced, suggesting that the mRNA sequence encoding yop $Q$ had been degraded. As a control, equal amounts of cat transcripts were amplified from yersiniae grown in the presence and absence of calcium. If the degradation of transcript requires the presence of $\mathrm{YopD} / \mathrm{LcrH}$, a $\Delta(y o p D Q)$ double mutant strain may be defective in reducing the yop $Q$ mRNA concentration. This was tested, and equal amounts of yopQ-npt, npt, and cat transcripts were found in the $\Delta(y o p D Q)$ strain grown in either the presence or absence of calcium. Thus, YopQ expression seems to be regulated by a mechanism that requires formation of the YopD-LcrH complex and that may be accompanied by the degradation of yop $Q$ mRNA.

YopD-LcrH complex binds yop $\boldsymbol{Q}$ mRNA. To test whether YopD and LcrH repress YopQ synthesis by binding to mRNA, recombinant proteins were purified and mixed with ${ }^{32} \mathrm{P}$-labeled yop $Q$ mRNA generated by in vitro transcription with $\mathrm{T} 7$ polymerase (Fig. 5). YopD/LcrH bound ${ }^{32} \mathrm{P}$-labeled yopQ mRNA, as indicated by mobility shifts of the RNA probe during polyacrylamide gel electrophoresis (Fig. 5A and B). RNA binding of YopD and LcrH appears to be specific for yop $Q$ transcripts, as mobility shifts were prevented by the addition of excess unlabeled yopQ mRNA. Although YopD and LcrH could also bind to $E$. coli tRNA, significantly greater amounts of the tRNA were required to displace YopD and $\mathrm{LcrH}$ from yopQ mRNA (Fig. 5F). LcrH alone did not bind ${ }^{32} \mathrm{P}$-labeled yop $Q$ mRNA (Fig. 5C and D). Expression of YopD alone in either $E$. coli or $Y$. enterocolitica caused aggregation and insolubility of this polypeptide in the bacterial cytoplasm (Fig. 5E).

Thus, formation of YopD-LcrH complexes seems to be a prerequisite for binding of these polypeptides to the yop $Q$ transcript. Multiple gel-shifted ${ }^{32} \mathrm{P}$-labeled yop $Q$ species were observed at higher concentrations of YopD and LcrH (Fig. 5B). This could be due to the binding of multiple YopD and LcrH molecules to each transcript. We calculated the concentration for $50 \%$ binding to ${ }^{32} \mathrm{P}$-labeled yop $Q$ mRNA as $8 \times 10^{-6} \mathrm{M}$ YopD and $\mathrm{LcrH}$. As the on and off rates of proteins for mRNA targets are affected by equilibrium changes during gel electrophoresis (11), this measurement must be considered an approximation of the affinity of YopD and LcrH for the yopQ transcript.

yopD mutations suppress synthesis and secretion defects of yop $Q$ signal mutants. Previous work mapped the yop $Q$ secretion signal to codons 1 to 15 . The +1 and -2 frameshift mutations of this element did not affect secretion signaling; however, the -1 frameshift mutation severely reduced reporter gene expression when type III machines were induced by low calcium (6). One explanation for this phenotype is that the yop $Q_{-1}$ mutation may be defective in secretion signaling, preventing initiation of the mutant transcripts into the type III pathway.

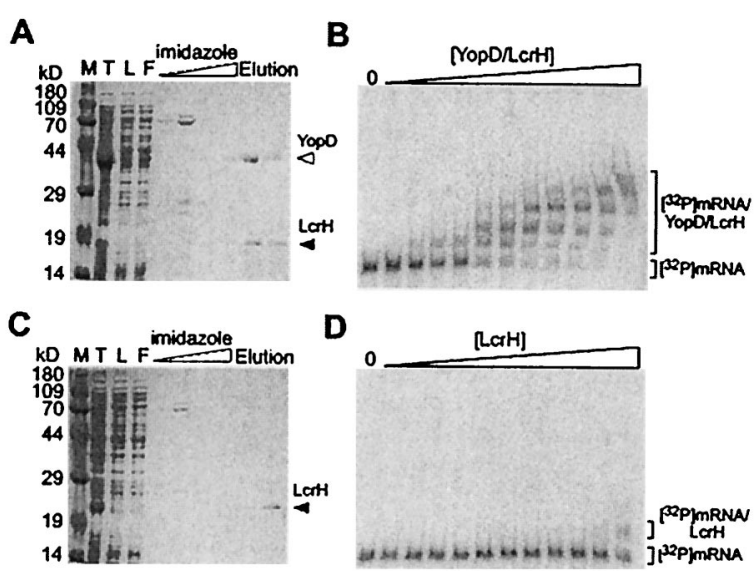

E

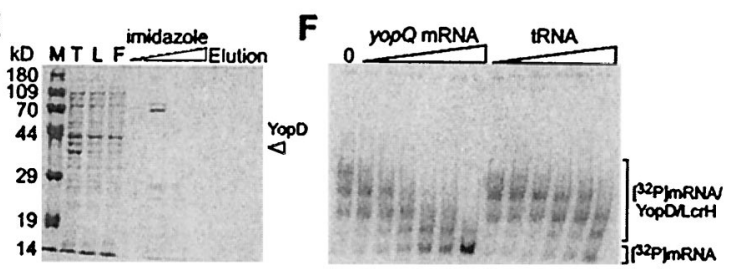

FIG. 5. YopD and LcrH complexes bind yopQ mRNA. A transcriptional yopD-lcrH fusion (A), lcrH (C), or yopD (E) was cloned into pET16 (Novagen) and transformed into E. coli BL21(DE3). Protein was expressed by IPTG induction of T7 polymerase. Total cell extracts (T) were centrifuged, and the cleared supernatant (L, load) was applied to affinity chromatography on Ni-NTA. Flowthrough (F), column wash samples with increasing stringency (imidazole), and elution with $0.5 \mathrm{M}$ imidazole were analyzed by separating proteins on SDS $-15 \%$ PAGE and staining with Coomassie blue. The migration of molecular size markers is indicated. ${ }^{32} \mathrm{P}$-labeled yop $Q$ mRNA was obtained by in vitro transcription of pDA340 with $\left[{ }^{32} \mathrm{P}\right] \mathrm{UTP}$ and gel purification. ${ }^{32} \mathrm{P}$-labeled yop $Q$ mRNA $(10 \mathrm{fmol})$ was incubated in the presence of increasing amounts of purified YopD and LcrH $(0,0.9,1.8,2.7,3.6$, 4.5, 5.4, 6.3, 7.2, 8.1, 9, and $27 \mathrm{ng}$ ) (B) or LcrH alone (D) and separated by electrophoresis on a $4 \%$ polyacrylamide gel. Specificity of yopQ mRNA binding ( $9 \mathrm{ng}$ of YopD and LcrH to $10 \mathrm{fmol}$ of ${ }^{32} \mathrm{P}$-labeled yop $Q$ mRNA) was assessed by adding excess unlabeled yop $Q$ or $E$. coli tRNA $(0,10,20,50,80,100$, and $500 \mathrm{fmol})(\mathrm{F})$.

To test whether yopD is required for the reduced synthesis of $\mathrm{YopQ}_{-1}-\mathrm{Npt}$, we examined reporter gene expression in various genetic backgrounds (Fig. 6). Wild-type yersiniae synthesized only small amounts of YopQ ${ }_{-1}-\mathrm{Npt}$, and the polypeptide remained in the bacterial cytoplasm. The $\Delta(y o p D)$ mutant strain synthesized 15 -fold more YopQ ${ }_{-1}-\mathrm{Npt}$, and $58 \%$ of the polypeptide was secreted into the extracellular medium, suggesting that the yopD knockout mutation suppressed both polypeptide synthesis and secretion defects caused by the yop $Q_{-1}$ mutation. $\Delta(y o p N)$ mutant yersiniae are known to increase virulence gene expression in the presence of calcium; however, the mutant cells were unable to increase expression or secretion of the YopQ ${ }_{-1}-\mathrm{Npt}$ fusion. Together these data suggest that YopD and LcrH prevent polypeptide synthesis of defective secretion signals, presumably by sequestration of unused transcripts and initiation into a degradation pathway.

\section{DISCUSSION}

Regulatory control of the Yersinia type III pathway was first revealed by the isolation of low-calcium response $(l c r)$ mutants 


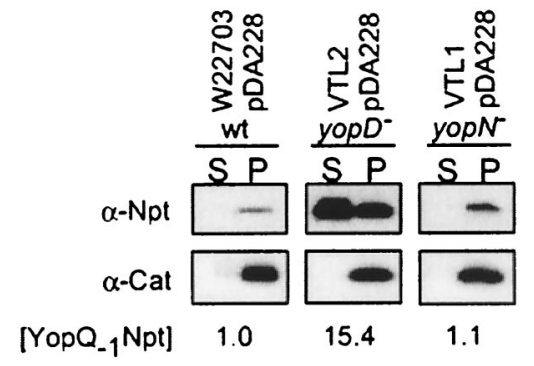

FIG. 6. yopD mutation suppresses the signaling defect of the YopQ $_{-1}$-Npt mutation. The secretion signal of yopQ, i.e., codons 1 to 15 fused to $n p t$, was mutated by deleting a nucleotide immediately following the AUG translational start. The reading frameshift was corrected at the fusion site with the $n p t$ reporter, generating the $\mathrm{YopQ}_{-1}$-Npt fusion (pDA228). Wild-type $Y$. enterocolitica W22703 (wt), the $\Delta($ yopN) mutant strain VTL1, and the $\Delta(y o p D)$ mutant VTL2 were transformed with pDA228. Cultures were induced for type III secretion by growing yersiniae at $37^{\circ} \mathrm{C}$ in the absence of calcium. Cultures were centrifuged, and the extracellular medium was separated into supernatant $(S)$ and bacterial pellet $(\mathrm{P})$. The expression and location of $\mathrm{YopQ}_{-1}$-Npt and cytoplasmic chloramphenicol acetyltransferase (Cat) was measured by immunoblotting with specific antibody. The secretion of YopQ ${ }_{-1}-\mathrm{Npt}$ was quantified with an alpha imager: W22703, 0\%; $\Delta(y o p D)$ mutant VTL2, 58\%; and the $\Delta(y o p N)$ mutant strain VTL1, $0 \%$. The relative concentration of the protein [YopQ $\left.{ }_{-1}-\mathrm{Npt}\right]$ is indicated for each strain.

(72). lcrE (yopN) mutants display a temperature-sensitive growth defect (71), as these strains secrete massive amounts of Yop proteins when grown at $37^{\circ} \mathrm{C}$ in the presence of calcium (23). Loss-of-function mutations in $s y c N$, tye $A$, or $y s c B$ cause a similar phenotype $(14,20,35,37,38)$. The products of the $l c r E$ $(y \circ p N), s y c N$, tye $A$, and $y s c B$ genes presumably block the type III secretion pathway in the presence of calcium (16). Mutations in $l c r H$ and yopD also affect calcium regulation of the Yersinia type III pathway. The growth defect of $l c r H$ and yopD mutants is not as severe as that of $l c r E(y o p N), s y c N, y s c B$, and tye $A$ strains $(56,62,72) . l c r H$ and yopD are expressed from the lcrGVHyopBD operon (8). Both genes have previously been implicated in regulating expression of the yop virulon $(8,70)$, but the mechanism of regulation was hitherto unknown.

We observed that $y o p N, \operatorname{syc} N$, ysc $B$, and tye $A$ mutations abolish the calcium regulation of YopQ synthesis and secretion (class I mutants). In contrast, yopD and $l c r H$ mutations abolish the regulation of YopQ synthesis without altering the regulation of YopQ secretion (class II mutants). It is suggested here that regulation of yop $Q$ expression occurs at the posttranscriptional level and is mediated by yopD and $l c r H$. YopD and LcrH bind directly to yop $Q$ transcript and could act as a repressor of translation. YopD and LcrH may bind to several different transcripts, as yopD and $l c r H$ mutations are known to affect the expression of yopE, yop $Q$, and yop $M$, among others $(8,70)$. We speculate that $\mathrm{YopD} / \mathrm{LcrH}$ may function as a repressor for the translation of transcripts that encode type III secretion substrates. When the type III pathway is inactive, this regulatory mechanism presumably represses the synthesis of Yop proteins and prevents the accumulation of polypeptides that cannot be secreted. Type III export of YopD may deplete the repressor complex from the bacterial cytoplasm, triggering recognition of yop transcripts by the secretion machinery. Thus, secretion of YopD can be viewed as a regulatory switch for the activation of the type III pathway.
It is conceivable that YopD and LcrH prevent the recognition of translational initiation signals by ribosomes and regulate translational initiation of yop $Q$ mRNA. If so, the binding site of YopD and LcrH could be located within the untranslated leader of yop $Q$ (nucleotides -178 through +3 relative to the AUG start codon), as fusion of this region to $n p t$ is sufficient for $y o p D / l c r H$-mediated regulation. Recent experiments suggest the possibility that additional genes are required for yopD- and $\mathrm{lcr} H$-mediated control of yop gene expression. Williams and Straley as well as Lee et al. suggest that yscM1 and $y s c M 2$ act at the same regulatory step of yop gene expression $(42,70)$. This is a surprising result, as LcrQ (YscM1 and YscM2) is believed to control the transcriptional regulation of yop expression $(54,61)$. Future work will need to unravel the molecular mechanism of yopD-, lcrH-, yscM1-, and yscM2-mediated regulation of yop expression.

\section{ACKNOWLEDGMENTS}

We thank Vincent Lee and Nghia Truong for help and reagents.

This work was supported by NIAID-NIH grant AI42797 to O.S. D.M.A. was supported by NIH training grant AI07323 and the Warsaw Family Fellowship. K.R. was supported by NIH training grant AI07323.

\section{REFERENCES}

1. Allaoui, A., R. Schulte, and G. R. Cornelis. 1995. Mutational analysis of the Yersinia enterocolitica virC operon: characterization of yscE, $F, G, I, J, K$ required for Yop secretion and $y s c H$ encoding YopR. Mol. Microbiol. 18: 343-355.

2. Allaoui, A., S. Woestyn, C. Sluiters, and G. Cornelis. 1994. YscU, a Yersinia enterocolitica inner membrane protein involved in Yop secretion. J. Bacteriol. 176:4534-4542.

3. Anderson, D. M., D. Fouts, A. Collmer, and O. Schneewind. 1999. Reciprocal secretion of proteins by the bacterial type III machines of plant and animal pathogens suggests universal recognition of mRNA targeting signals. Proc. Natl. Acad. Sci. USA 96:12839-12843.

4. Anderson, D. M., and O. Schneewind. 1997. A mRNA signal for the type III secretion of Yop proteins by Yersinia enterocolitica. Science 278:1140-1143.

5. Anderson, D. M., and O. Schneewind. 1999. Type III machines of Gramnegative pathogens: injecting virulence factors into host cells and more. Curr. Opin. Microbiol. 2:18-24.

6. Anderson, D. M., and O. Schneewind. 1999. Yersinia enterocolitica type III secretion: an mRNA signal that couples translation and secretion of YopQ. Mol. Microbiol. 31:1139-1148.

7. Bergmann, T., K. Erickson, E. Galyov, C. Persson, and H. Wolf-Watz. 1994. The $l c r B(y s c N / U)$ gene cluster of Yersinia pseudotuberculosis is involved in Yop secretion and shows high homology to the spa gene clusters of Shigella flexneri and Salmonella typhimurium. J. Bacteriol. 176:2619-2626.

8. Bergmann, T., S. Hakansson, A. Forsberg, L. Norlander, A. Macellaro, A. Backman, I. Bolin, and H. Wolf-Watz. 1991. Analysis of V antigen lcrGVHyopBD operon of Yersinia pseudotuberculosis: evidence for a regulatory role of $l c r H$ and $l c r V$. J. Bacteriol. 173:1607-1616.

9. Boland, A., M.-P. Sory, M. Iriarte, C. Kerbourch, P. Wattiau, and G. R. Cornelis. 1996. Status of YopM and YopN in the Yersinia yop virulon: YopM of $Y$. enterocolitica is internalized inside the cytosol of PU5-1.8 macrophages by the YopB, D, N delivery apparatus. EMBO J. 15:5191-5201.

10. Cambronne, E. D., L. W. Cheng, and O. Schneewind. 2000. LcrQ/YscM1, regulators of the Yersinia yop virulon, are injected into host cells by a chaperone dependent mechanism. Mol. Microbiol. 37:263-273.

11. Carey, J., V. Cameron, P. L. de Haseth, and O. C. Uhlenbeck. 1983. Sequence-specific interaction of R17 coat protein with its ribonucleic acid binding site. Biochemistry 22:2601-2610.

12. Cheng, L. W., D. M. Anderson, and O. Schneewind. 1997. Two independent type III secretion mechanisms for YopE in Yersinia enterocolitica. Mol. Microbiol. 24:757-765.

13. Cheng, L. W., O. Kay, and O. Schneewind. 2001. Regulated secretion of YopN by the type III machinery of Yersinia enterocolitica. J. Bacteriol. 183: 5293-5301.

14. Cheng, L. W., and O. Schneewind. 2000. Yersinia enterocolitica TyeA, an intracellular regulator of the type III machinery, is required for the specific targeting of YopE, YopH, YopM, and YopN into the cytosol of eukaryotic cells. J. Bacteriol. 182:3183-3190. 
15. Cheng, L. W., and O. Schneewind. 1999. Yersinia enterocolitica type III secretion: On the role of SycE in targeting YopE into HeLa cells. J. Biol. Chem. 274:22102-22108.

16. Cornelis, G. R. 1998. The Yersinia deadly kiss. J. Bacteriol. 180:5495-5504.

17. Cornelis, G. R., A. Boland, A. P. Boyd, C. Geuijen, M. Iriarte, C. Neyt, M.-P. Sory, and I. Stainier. 1998. The virulence plasmid of Yersinia, an antihost genome. Microbiol. Mol. Biol. Rev. 62:1315-1352.

18. Cornelis, G. R., and C. Colson. 1975. Restriction of DNA in Yersinia enterocolitica detected by the recipient ability for a derepressed $\mathrm{R}$ factor from Escherichia coli. J. Gen. Microbiol. 87:285-291.

19. Cornelis, G. R., and H. Wolf-Watz. 1997. The Yersinia yop virulon: a bacterial system for subverting eukaryotic cells. Mol. Microbiol. 23:861-867.

20. Day, J. B., and G. V. Plano. 1998. A complex composed of SycN and YscB functions as a specific chaperone for YopN in Yersinia pestis. Mol. Microbiol. 30:777-789.

21. Fallman, M., K. Andersson, S. Hakansson, K. E. Magnusson, O. Stendahl, and H. Wolf-Watz. 1995. Yersinia pseudotuberculosis inhibits Fc receptormediated phagocytosis in J774 cells. Infect. Immun. 63:3117-3124.

22. Fields, K. A., and S. C. Straley. 1999. LcrV of Yersinia pestis enters infected eukaryotic cells by a virulence plasmid-independent mechanism. Infect. Immun. 67:4801-4813.

23. Forsberg, A., A.-M. Viitanen, M. Skunik, and H. Wolf-Watz. 1991. The surface-located YopN protein is involved in calcium signal transduction in Yersinia pseudotuberculosis. Mol. Microbiol. 5:977-986.

24. Goguen, J. D., J. Yother, and S. C. Straley. 1984. Genetic analysis of the low calcium response in Yersinia pestis Mud1(Ap lac) insertion mutants. J. Bacteriol. 160:842-848.

25. Gold, L., D. Pribnow, T. Schneider, S. Shinedling, B. S. Singer, and G. Stormo. 1981. Translational initiation in prokaryotes. Annu. Rev. Microbiol. 35:365-403.

26. Gottesman, M., A. Oppenheim, and D. Court. 1982. Retroregulation: control of gene expression from sites distal to the gene. Cell 29:727-728.

27. Hakansson, S., T. Bergman, J.-C. Vanooteghem, G. Cornelis, and H. WolfWatz. 1993. YopB and YopD constitute a novel class of Yersinia Yop proteins. Infect. Immun. 61:71-80.

28. Hakansson, S., E. Gaylov, R. Rosqvist, and H. Wolf-Watz. 1996. The Yersinia YpkA Ser/Thr kinase is translocated and subsequently targeted to the inner surface of the HeLa cell plasma membrane. Mol. Microbiol. 20:593603.

29. Hakansson, S., K. Schesser, C. Persson, E. E. Galyov, R. Rosqvist, F Homble, and H. Wolf-Watz. 1996. The YopB protein of Yersinia pseudotuberculosis is essential for the translocation of Yop effector proteins across the target cell plasma membrane and displays a contact-dependent membrane disrupting activity. EMBO J. 15:5812-5823.

30. Ham, J. H., D. W. Bauer, D. E. Fouts, and A. Collmer. 1998. A cloned Erwinia chrysanthemi Hrp (type III protein secretion) system functions in Escherichia coli to deliver Pseudomonas syringae Avr signals to plant cells and to secrete Avr proteins in culture. Proc. Natl. Acad. Sci. USA 95:1020610211 .

31. Hoe, N. P., F. C. Minion, and J. D. Goguen. 1992. Temperature sensing in Yersinia pestis: regulation of yopE transcription by $l c r F$. J. Bacteriol. 174: 4275-4286.

32. Hoiczyk, E., and G. Blobel. 2001. Polymerization of a single protein of the pathogen Yersinia enterocolitica into needles punctures eukaryotic cells. Proc. Natl. Acad. Sci. USA 98:4669-4674.

33. Holmstrom, A., J. Olsson, P. Cherepanov, E. Maier, R. Nordfelth, J. Petterson, R. Benz, H. Wolf-Watz, and A. Forsberg. 2001. LcrV is a channel size-determining component of the Yop effector translocon of Yersinia. Mol. Microbiol. 39:620-632.

34. Hueck, C. J. 1998. Type III protein secretion in bacterial pathogens of animals and plants. Microbiol. Mol. Biol. Rev. 62:379-433.

35. Iriarte, M., and G. R. Cornelis. 1999. Identification of SycN, YscX, and YscY, three new elements of the Yersinia yop virulon. J. Bacteriol. 181:675680.

36. Iriarte, M., and G. R. Cornelis. 1998. YopT, a new Yersinia effector protein, affects the cytoskeleton of host cells. Mol. Microbiol. 29:915-929.

37. Iriarte, M., M.-P. Sory, A. Boland, A. P. Boyd, S. D. Mills, I. Lambermont, and G. R. Cornelis. 1998. TyeA, a protein involved in control of Yop release and in translocation of Yersinia Yop effectors. EMBO J. 17:1907-1918.

38. Jackson, M. W., J. B. Day, and G. V. Plano. 1998. YscB of Yersinia pestis functions as a specific chaperone for YopN. J. Bacteriol. 180:49124921.

39. Karlinsey, J. E., J. Lonner, K. L. Brown, and K. T. Hughes. 2000. Translation/secretion coupling by type III secretion systems. Cell 102:487-497.

40. Kubori, T., Y. Matsushima, D. Nakamura, J. Uralil, M. Lara-Tejero, A. Sukhan, J. E. Galan, and S.-I. Aizawa. 1998. Supermolecular structure of the Salmonella typhimurium type III protein secretion system. Science 280:602605.

41. Lee, V. T., D. M. Anderson, and O. Schneewind. 1998. Targeting of Yersinia Yop proteins into the cytosol of HeLa cells: one-step translocation of YopE across bacterial and eukaryotic membranes is dependent on SycE chaperone. Mol. Microbiol. 28:593-601.
42. Lee, V. T., S. K. Mazmanian, and O. Schneewind. 2001. A program of Yersinia enterocolitica type III secretion reactions is triggered by specific host signals. J. Bacteriol. 183:4970-4978.

43. Lee, V. T., and O. Schneewind. 2001. Protein secretion and the pathogenesis of bacterial infections. Genes Dev. 15:1725-1752.

44. Lee, V. T., and O. Schneewind. 1999. Type III machines of pathogenic yersiniae secrete virulence factors into the extracellular milieu. Mol. Microbiol. 31:1619-1629.

45. Lee, V. T., C. Tam, and O. Schneewind. 2000. Yersinia enterocolitica type III secretion. LcrV, a substrate for type III secretion, is required for toxin-targeting into the cytosol of HeLa cells. J. Biol. Chem. 275:3686936875 .

46. Lloyd, S. A., A. Forsberg, H. Wolf-Watz, and M. S. Francis. 2001. Targeting exported substrates to the Yersinia TTSS: different functions for different signals? Trends Microbiol. 9:367-371.

47. Lloyd, S. A., M. Norman, R. Rosqvist, and H. Wolf-Watz. 2001. Yersinia YopE is targeted for type III secretion by N-terminal, not mRNA, signals. Mol. Microbiol. 39:520-531.

48. Miller, J. H., and A. M. Albertini. 1983. Effects of surrounding sequence on the suppression of nonsense codons. J. Mol. Biol. 164:59-71.

49. Mills, S. D., A. Boland, M.-P. Sory, P. van der Smissen, C. Kerbouch, B. B. Finlay, and G. R. Cornelis. 1997. Yersinia enterocolitica induces apoptosis in macrophages by a process requiring functional type III secretion and translocation mechanisms and involving YopP, presumably acting as an effector protein. Proc. Natl. Acad. Sci. USA 94:12638-12643.

50. Monack, D. M., J. Mecsas, N. Ghori, and S. Falkow. 1997. Yersinia signals macrophages to undergo apoptosis and YopJ is necessary for this cell death. Proc. Natl. Acad. Sci. USA 94:10385-10390.

51. Persson, C., N. Carballeira, H. Wolf-Watz, and M. Fallman. 1997. The PTPase YopH inhibits uptake of Yersinia, tyrosine phosphorylation of $\mathrm{p} 130^{\mathrm{cas}}$ and FAK, and the associated accumulation of these proteins in peripheral focal adhesion. EMBO J. 16:2307-2318.

52. Persson, C., R. Nordfelth, A. Holmstrom, S. Hakansson, R. Rosqvist, and H. Wolf-Watz. 1995. Cell-surface-bound Yersinia translocate the protein tyrosine phosphatase YopH by a polarized mechanism into the target cell. Mol. Microbiol. 18:135-150.

53. Petterson, J., A. Holmstrom, J. Hill, E. Frithz-Lindsten, A. von Euler-Matell, E. Carlsson, R. Titball, A. Forsberg, and H. Wolf-Watz. 1999. The V-antigen of Yersinia is surface exposed before target cell contact and involved in virulence protein translocation. Mol. Microbiol. 32:961-976.

54. Petterson, J., R. Nordfelth, E. Dubinina, T. Bergman, M. Gustafsson, K. E. Magnusson, and H. Wolf-Watz. 1996. Modulation of virulence factor expression by pathogen target cell contact. Science 273:1231-1233.

55. Price, S. B., C. Cowan, R. D. Perry, and S. C. Straley. 1991. The Yersinia pestis $\mathrm{V}$ antigen is a regulatory protein necessary for $\mathrm{Ca}^{2+}$-dependent growth and maximal expression of low- $\mathrm{Ca}^{2+}$ response virulence genes. J. Bacteriol. 173:2649-2657.

56. Price, S. B., and S. C. Straley. 1989. lcrH, a gene necessary for virulence of Yersinia pestis and for the normal response of $Y$. pestis to ATP and calcium. Infect. Immun. 57:1491-1498.

57. Reiss, B., R. Sprengel, and H. Schaller. 1984. Protein fusions with the kanamycin resistance gene from transposon Tn 5. EMBO J. 3:33173322 .

58. Rimpilainen, M., A. Forsberg, and H. Wolf-Watz. 1992. A novel protein, LcrQ, involved in the low-calcium response of Yersinia pseudotuberculosis shows extensive homology to YopH. J. Bacteriol. 174:3355-3363.

59. Rosqvist, R., K.-E. Magnusson, and H. Wolf-Watz. 1994. Target cell contact triggers expression and polarized transfer of Yersinia YopE cytotoxin into mammalian cells. EMBO J. 13:964-972.

60. Sory, M.-P., and G. R. Cornelis. 1994. Translocation of a hybrid YopEadenylate cyclase from Yersinia enterocolitica into HeLa cells. Mol. Microbiol. 14:583-594.

61. Stainier, I., M. Iriarte, and G. R. Cornelis. 1997. YscM1 and YscM2, two Yersinia enterocolitica proteins causing downregulation of yop transcription. Mol. Microbiol. 26:833-843.

62. Straley, S. C., G. V. Plano, E. Skrzypek, P. L. Haddix, and K. A. Fields. 1993. Regulation by $\mathrm{Ca}^{2+}$ in the Yersinia low- $\mathrm{Ca}^{2+}$ response. Mol. Microbiol. 8:1005-1010.

63. Studier, F. W., A. H. Rosenberg, J. J. Dunn, and J. W. Dubendorff. 1990. Use of T7 polymerase to direct expression of cloned genes. Methods Enzymol. 185:60-89.

64. Takeshita, S., M. Sato, M. Toba, W. Masahashi, and T. Hashimoto-Gotoh. 1987. High-copy-number and low-copy-number plasmid vectors for LacZ alpha-complementation and chloramphenicol- or kanamycin-resistance selection. Gene 61:63-74.

65. Tardy, F., F. Homble, C. Neyt, R. Wattiez, G. R. Cornelis, J.-M. Ruysschaert, and V. Cabiaux. 1999. Yersinia enterocolitica type III secretiontranslocation system: channel formation by secreted Yops. EMBO J. 18:6793-6799.

66. Wattiau, P., B. Bernier, P. Deslee, T. Michiels, and G. R. Cornelis. 1994. Individual chaperones required for Yop secretion by Yersinia. Proc. Natl. Acad. Sci. USA 91:10493-10497. 
67. Wattiau, P., and G. R. Cornelis. 1994. Identification of DNA sequences recognized by VirF, the transcriptional activator of the Yersinia yop regulon. J. Bacteriol. 176:3878-3884.

68. Wattiau, P., and G. R. Cornelis. 1993. SycE, a chaperone-like protein of Yersinia enterocolitica involved in the secretion of YopE. Mol. Microbiol. 8:123-131.

69. Wattiau, P., S. Woestyn, and G. R. Cornelis. 1996. Customized secretion chaperones in pathogenic bacteria. Mol. Microbiol. 20:255-262.
70. Williams, A. W., and S. C. Straley. 1998. YopD of Yersinia pestis plays a role in negative regulation of the low-calcium response in addition to its role in translocation of Yops. J. Bacteriol. 180:350-358.

71. Yother, J., T. W. Chamness, and J. D. Goguen. 1986. Temperature-controlled plasmid regulon associated with low calcium response in Yersinia pestis. J. Bacteriol. 165:443-447.

72. Yother, J., and J. D. Goguen. 1985. Isolation and characterization of $\mathrm{Ca}^{2+}$ blind mutants of Yersinia pestis. J. Bacteriol. 164:704-711. 